\title{
Sleep-dependent learning and motor-skill complexity
}

\author{
Kenichi Kuriyama, ${ }^{1,2}$ Robert Stickgold, ${ }^{1}$ and Matthew P. Walker ${ }^{1,3,4}$ \\ ${ }^{1}$ Center for Sleep and Cognition, Department of Psychiatry, Harvard Medical School, Beth Israel Deaconess Medical Center, \\ Boston Massachusetts 02215, USA; ${ }^{2}$ Department of Psychophysiology, National Institute of Mental Health, National Center of \\ Neurology and Psychiatry, Kohnodai, Ichikawa 272-0827, Japan; ${ }^{3}$ Sleep and Neuroimaging Laboratory, Department of \\ Psychiatry, Harvard Medical School, Beth Israel Deaconess Medical Center, Boston Massachusetts 02215, USA
}

\begin{abstract}
Learning of a procedural motor-skill task is known to progress through a series of unique memory stages. Performance initially improves during training, and continues to improve, without further rehearsal, across subsequent periods of sleep. Here, we investigate how this delayed sleep-dependent learning is affected when the task characteristics are varied across several degrees of difficulty, and whether this improvement differentially enhances individual transitions of the motor-sequence pattern being learned. We report that subjects show similar overnight improvements in speed whether learning a five-element unimanual sequence (17.7\% improvement), a nine-element unimanual sequence $(20.2 \%)$, or a five-element bimanual sequence $(17.5 \%)$, but show markedly increased overnight improvement $(28.9 \%)$ with a nine-element bimanual sequence. In addition, individual transitions within the motor-sequence pattern that appeared most difficult at the end of training showed a significant $17.8 \%$ increase in speed overnight, whereas those transitions that were performed most rapidly at the end of training showed only a non-significant $1.4 \%$ improvement. Together, these findings suggest that the sleep-dependent learning process selectively provides maximum benefit to motor-skill procedures that proved to be most difficult prior to sleep.
\end{abstract}

A growing literature in recent years argues that sleep plays a crucial role in learning and memory consolidation (for reviews, see Smith 1995; Stickgold et al. 2001; Walker 2004). Perhaps the most consistent evidence has been for sleep's role in the development of procedural skill memory. Specifically, sleep has been implicated in the ongoing process of consolidation after initial acquisition, whereby delayed learning is achieved in the absence of further practice. Evidence of sleep-dependent procedural skill learning has now been demonstrated across a wide variety of skill domains, including visual (Karni et al. 1994; Gais et al. 2000; Stickgold et al. 2000a,b), auditory (Atienza et al. 2004; Gaab et al. 2004), and motor (Smith and MacNeill 1994; Fischer et al. 2002; Walker et al. 2002, 2003a,b; Korman et al. 2003; Robertson et al. 2004) systems.

Regarding motor-sequence learning, Walker et al. (2002) have shown that a night of sleep can trigger significant performance improvements in speed and accuracy on a finger-tapping task, whereas equivalent periods of time during wake provided no significant benefit. Furthermore, these overnight learning gains correlated with the amount of stage-2 NREM sleep, particularly late in the night. It was also shown that three nights of sleep provide larger benefits for motor-skill performance than does one night, and that the mechanisms of sleep-dependent learning may be dissociable from those governing both initial practicedependent learning during training (Walker et al. 2003b) and performance stabilization across periods of wake (Walker et al. 2003a). Using the same task, Fischer et al. (2002) have shown that sleep on the first night following training is crucial for this delayed improvement to develop, and that $8 \mathrm{~h}$ of sleep during the day triggers improvements similar to those achieved following nocturnal sleep. This report, however, described a correlation with REM sleep and not stage-2 NREM.

Little is known, however, about the details of this sleepdependent learning or its generality. For example, although there

\footnotetext{
${ }^{4}$ Corresponding author.

E-mail mwalker@hms.harvard.edu; fax (617) 667-8498.

Article and publication are at http://www.learnmem.org/cgi/doi/10.1101/ Im.76304.
}

appears to be no transfer of this sleep-dependent learning to new motor sequences or to performance by the other hand (Fischer et al. 2002; Korman et al. 2003), it is not clear whether sleepdependent learning occurs when the trained sequence requires bimanual performance or when the sequence becomes longer and more complex. Considering that the majority of motor skills we acquire across the life span are of an intricate and often multidigit and multilimb nature, it becomes particularly germane to understand potential differences in sleep-dependent learning across a spectrum of motor-skill difficulty. Similarly, it is not known which aspects of the motor sequence are actually enhanced by sleep. When one initially learns a complex motor sequence, it is common for there to be transition points that are harder and others that are easier, and it is unclear whether sleep benefits all of these transitions or can selectively enhance either the easier or harder components of the sequence.

In the current study, we begin to address these issues. First, we investigate sleep-dependent motor-skill learning by varying two characteristics of the finger-tapping task as follows: (1) the limb complexity-either unimanual or bimanual, and (2) the sequence length-either five-elements or nine-elements. In the current report, we focus primarily on differences in delayed, overnight improvement in task performance. Because increasing the sequence length or using a bimanual versus unimanual technique can alter the underlying neural systems recruited during initial training-dependent performance (e.g., Andres et al. 1999; Gerloff and Andres 2002; Grafton et al. 2002; Muller et al. 2002; De Weerd et al. 2003), we hypothesized that as task complexity increased, and thus the magnitude of information to be learned was greater, so too would the ensuing overnight benefit that sleep could provide. Secondly, on the basis of previous observations that increased motor-skill proficiency is associated with improved temporal automaticity between key-press transitions (Miller 1956; Rosenbaum et al. 1983; Sakai et al. 2003), we further hypothesized that there would be significant changes in the motor-sequence performance profile after a night of sleep, and specifically, that the slowest transition points of the sequence (those that were most difficult) would be selectively enhanced. To assess 

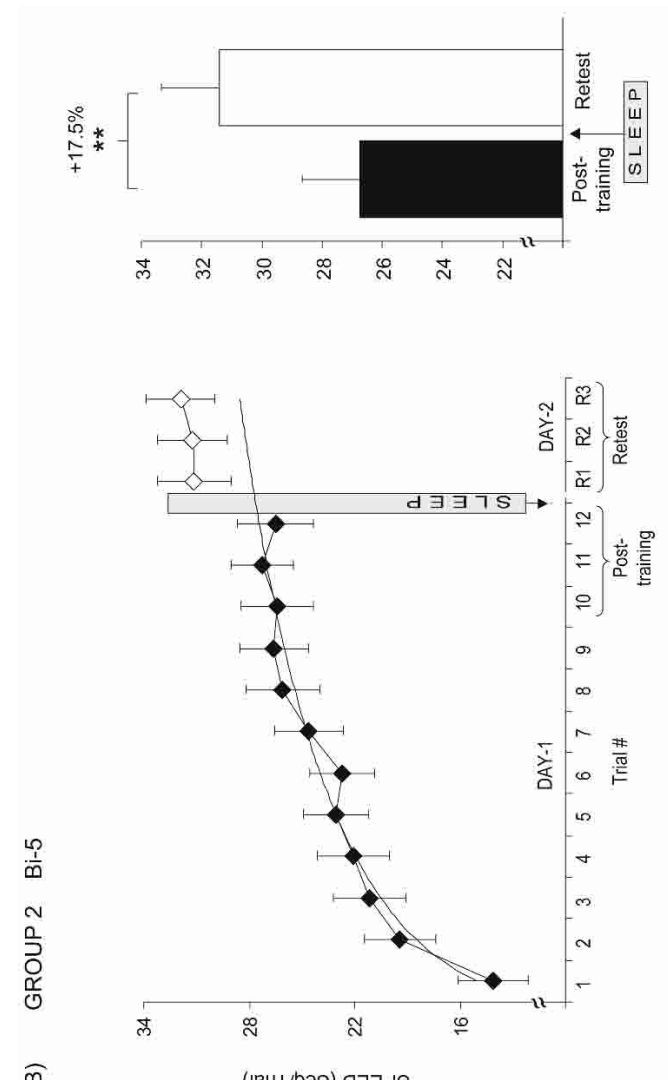

ติ

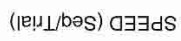

$\frac{1}{0}$
$\frac{1}{0}$
0
$\frac{0}{0}$
$\frac{0}{0}$

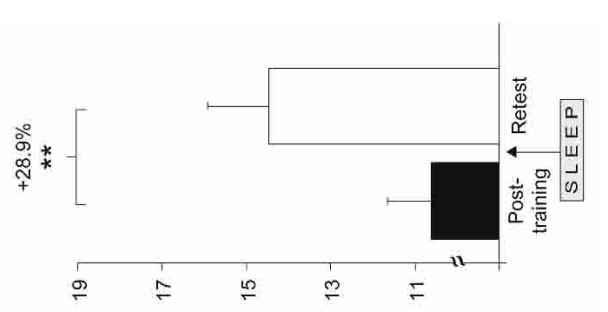

을 원

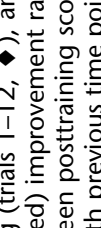

원

$\stackrel{\circ}{\longrightarrow}=$

ธิ

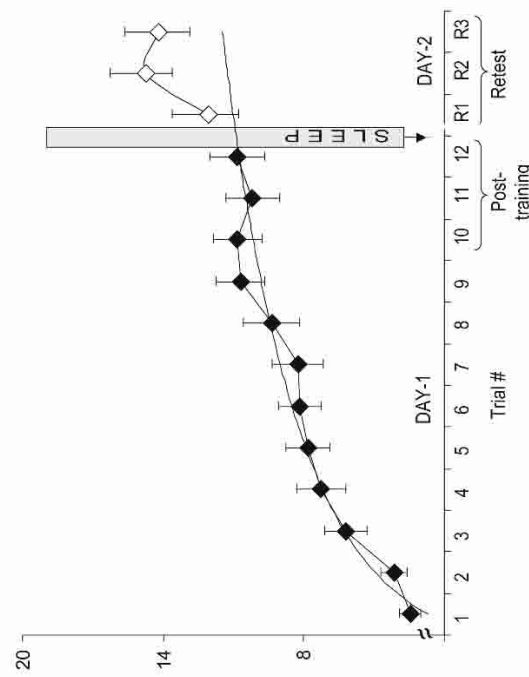

돈 은은

政

월 है

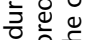

ษั

है.

巡

워워ํㄴ

등은

娄

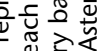

气ิ

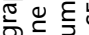

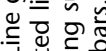

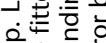

원응

오승

일 0

(घ! $\perp /$ bes $) 0 \exists \exists d S$

응 홍

읻ㅎํ. 는

过焉吉

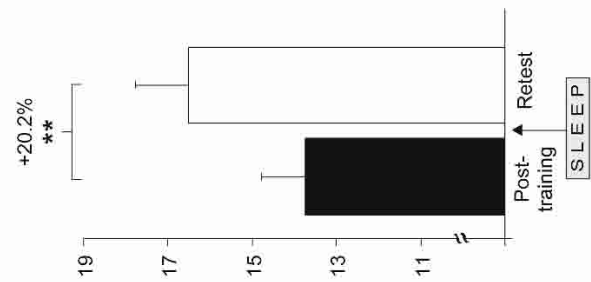

我

宅 论

产芦芯

듣등

次产 。

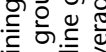

정

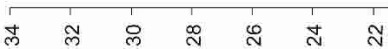

ช ญ

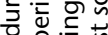

Y 这这选

은은

힣픈

ธับ응

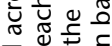

ญ흐응

0
0
$\frac{1}{5}$
$\frac{1}{5}$
0
0
0
$\frac{8}{0}$
0
0

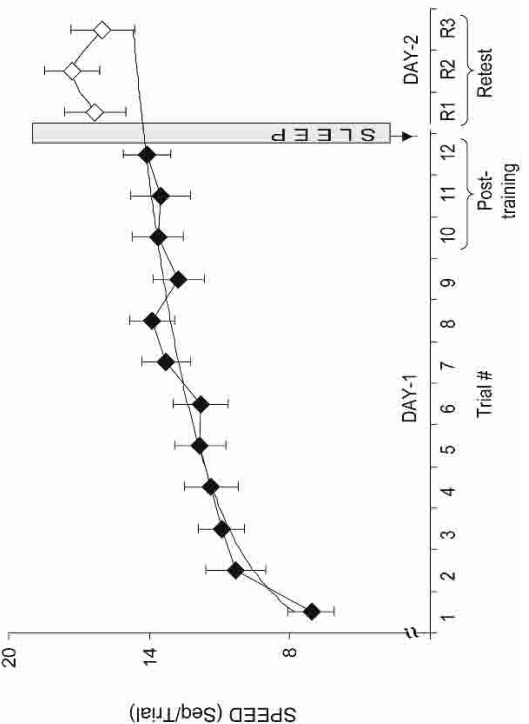

光万放

咅的祝

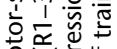

뜽엉

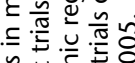

屯ै

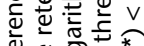

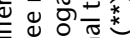

0 运落

-

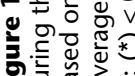


this possibility, we analyzed differences in transition speeds between each of the separate key-press movements within each sequence, before and after sleep. For example, in a unimanual five-element sequence, such as 4-1-3-2-4, where there are four key-press transitions, (a) from 4 to 1 , (b) from 1 to 3, (c) from 3 to 2 , and, finally, (d) from 2 to 4 , do pre- to post-sleep changes in the speeds of these transitions develop overnight (see Materials and Methods and Fig. 4A-D, below)?

\section{Results}

Subjects in all groups showed significant improvement in speed both during training and across a night of sleep, and significant overnight reductions in error rate.

\section{Group 1 (unimanual five-element task)}

Subjects in Group 1 primarily served as a reference for Groups $2-4$, performing the simplest form of the task utilizing four fingers (Uni-5), as used in our previous reports (Walker et al. 2002, 2003a,b).

\section{Practice-dependent learning}

During the initial training session on Day 1, performance speed improved $40.0 \%$ from Trial $1(16.3 \mathrm{seq} /$ trial $)$ to Trial $12[22.8$ seq/trial; $\mathrm{t}_{(14)}=5.08, P=0.0001 ;$ Fig. $\left.1 \mathrm{~A}\right]$. There was also a $33.9 \%$ decrease in error rate (improvement in accuracy), from 0.24 to 0.16 errors/seq, although this improvement was not significant $\left[\mathrm{t}_{(14)}=0.643, P=0.53\right]$.

\section{Overnight, sleep-dependent learning}

Following a night of sleep, retest performance indicated a significant $17.7 \%$ overnight improvement in speed $\left[\mathrm{t}_{(14)}=6.23\right.$, $P<0.0001$ ], from $22.7 \mathrm{seq} /$ trial posttraining (i.e., average of last three trials of Day 1) to $26.68 \mathrm{seq} /$ trial at retest (average of three trials on Day 2) (Fig.1A). Furthermore, this increase in speed was accompanied by a significant $47.8 \%$ reduction in error rate $\left[\mathrm{t}_{(14)}=3.73, P=0.002\right]$, from 0.15 to 0.08 errors $/ \mathrm{seq}$ (retest, Day 2).

\section{Group 2 (bimanual five-element task)}

In Group 2, we maintained the difficulty of the sequence length at five elements, but increased the task complexity by imposing the need for coordinated movements of the two hands. Subjects were again trained on Day 1 and retested after a night of sleep on Day 2. Note that as with the Uni-5 task, the Bi-5 task still required the use of only four fingers; but now two from each hand.

\section{Practice-dependent learning}

Across the training session, performance speed improved by $87.7 \%$, from 14.1 to $26.5 \mathrm{seq} /$ trial $\left[\mathrm{t}_{(14)}=5.99, P<0.0001\right.$, Fig.1B]. There was also a $61.3 \%$ decrease in error rate, from 0.46 to 0.18 errors/seq, but again, this difference was not significant $\left[\mathrm{t}_{(14)}=1.43, P=0.174\right]$.

\section{Overnight, sleep-dependent learning}

When retested after a night of sleep, performance showed a significant $17.5 \%$ improvement in speed, from 26.7 to $31.4 \mathrm{seq} /$ trial $\left[\mathrm{t}_{(14)}=7.22, P<0.0001\right.$, Fig.1B]. In addition, there was a significant $46.8 \%$ reduction in error rate, from 0.14 to 0.07 errors/seq [retest, Day 2; $\left.\mathrm{t}_{(14)}=3.38, P=0.004\right]$.

\section{Group 3: Unimanual nine-element task}

In contrast to Group 2, subjects in Group 3 were not required to perform cooperative movements between hands, but performed the task unimanually, as in Group 1. However, the task complexity was now increased on a different axis; the length of the sequence was now increased to nine elements. Note that even though the sequence length had increased relative to Groups 1 and 2, the task still required the use of only four fingers, as with Groups 1 and 2.

\section{Practice-dependent learning}

During the training session, a $99.0 \%$ improvement in performance speed, from 7.1 to $14.1 \mathrm{seq} /$ trial $\left[\mathrm{t}_{(12)}=10.93, P<0.0001\right.$, Fig.1C]. There was a $36.0 \%$ decrease in error rate, from 0.37 to 0.23 errors/seq, and again, the difference was not significant $\left[\mathrm{t}_{(12)}=1.74, P=0.106\right]$.

\section{Overnight, sleep-dependent learning}

At retest following a night of sleep, performance speed showed a $20.2 \%$ improvement, increasing from 13.8 to $16.5 \mathrm{seq} /$ trial $\left[\mathrm{t}_{(12)}=5.21, P=0.0002\right.$, Fig. $\left.1 \mathrm{C}\right]$. Together with this increase in speed came a $44.6 \%$ decrease in error rate, from 0.23 to 0.12 errors/seq [retest, Day 2; $\mathrm{t}_{(12)}=2.15, P=0.027$ ].

\section{Group 4: Bimanual nine-element task}

In Group 4, we increased the difficulty along both axes, with subjects required not only to perform a longer nine-element sequence (as in Group 3), but also to execute this longer sequence using cooperative movements of the two hands, as opposed to using just one hand. In this configuration, subjects were required to use eight fingers, as opposed to the four fingers used by the other groups.

\section{Practice-dependent learning}

Across the 12 training trials, performance speed showed a $213 \%$ increase, from 3.4 to $10.8 \mathrm{seq} /$ trial $\left[\mathrm{t}_{(13)}=7.65, P<0.0001\right.$, Fig.1D; two of the 14 subjects did not achieve any complete sequences on the first trial, and were therefore omitted from analysis]. Together with the improved speed, subjects' error rates decreased significantly by $62.2 \%$, from 1.10 to 0.42 errors/seq $\left[\mathrm{t}_{(13)}=2.93, P=0.013\right]$.

\section{Overnight, sleep-dependent learning}

After the night of sleep, performance speed showed a particularly large $28.9 \%$ improvement, increasing from 10.61 to $13.7 \mathrm{seq} /$ trial $\left[\mathrm{t}_{(13)}=4.45, P<0.0001 ;\right.$ Fig.1D]. The night of sleep also led to a $29.9 \%$ reduction in error rate, from 0.37 to 0.26 errors/seq $\left[\mathrm{t}_{(13)}=2.13, P=0.05\right]$. Closer examination of the post-sleep performance of Group 4 (Fig. 1D) revealed that the percentage improvement in performance speed (relative to posttraining) differed significantly across the three retest trials [ANOVA; $\left.F_{(2,39)}=3.58, P=0.037\right]$, with an overnight improvement of $14.2 \%$ on the first retest trial, but significantly larger improvements on the second and third retest trials, of $39.8 \%\left[\mathrm{t}_{(13)}=4.34\right.$, $P<0.001]$ and $35.0 \%\left[\mathrm{t}_{(13)}=2.26, P=0.041\right]$, respectively. This difference across retest trials was not present in any of the other three groups ( $P>0.32$ for each). It therefore appears that subjects require one trial before being able to express full delayed improvement in the most complex task configuration. When delayed learning was calculated on the basis of the second and third retest trials, the amount of overnight improvement in Group 4 was $36.4 \%$ [10.61 seq/trial posttraining Day 1 vs. $14.47 \mathrm{seq} /$ trial on Day $2\left(\mathrm{t}_{(13)}=5.78, P<0.0001\right]$, rather than the $28.9 \%$ obtained when averaging across all three retest trials.

On the basis of this analysis of the second and third retest trials, the overnight improvement in this group was more than twice that with the unimanual and bimanual five-element tasks. However, it was conceivable that the most complex task configuration simply was showing further practice-dependent improvement, or that delayed learning was occurring across wake as well as sleep in this more complex task. However, neither was found to be the case. When an additional group of subjects $(n=14$; matched in age and gender) was trained on the bimanual nine- 
element task at $11 \mathrm{am}( \pm 1 \mathrm{~h})$ and retested after an 8-h period of wake (a period equivalent to what subjects slept in Group 4), no significant improvement in either performance speed [11.4 seq/ trial posttraining vs. $11.5 \mathrm{seq} /$ trial retest; $0.9 \%$ improvement, $\left.\mathrm{t}_{(13)}=0.35, P=0.72\right]$, or accuracy [0.24 errors/seq posttraining vs. 0.25 errors/seq retest; $4.2 \%$ deterioration, $\mathrm{t}_{(13)}=0.51, P=0.61$ ) was observed. Therefore, the larger overnight improvements in performance on the most complex Bi-9 task version appear to reflect a specific sleep-dependent advantage, rather than an interaction of task difficulty and rehearsal or time per se.

\section{Performance differences between groups}

Although performance improved during initial training for each group individually, the percentage of practice-dependent improvement from the first to the last trial showed no significant difference across groups; either in performance speed [ANOVA; $F_{(3,53)}=0.92, P=0.43$ ], or accuracy [ANOVA; $F_{(3,53)}=1.95$, $P=0.13]$.

Whereas all groups then went on to improve significantly overnight, it was evident that the most complex version of the task, the Bi-9 configuration, demonstrated 50\% greater speed improvements relative to the Uni-5, Bi-5, and Uni-9 configurations ( $28.9 \%$ vs. a mean of $18.5 \%)$. When the overnight improvement seen on the last two retest trials in the Bi-9 group was compared with that in the other groups, significant group differences were seen, regardless of whether the Bi-9 group data were compared with all three trials of the other groups $\left[F_{(3,53)}=4.02, P=0.012\right]$ or to just the last two trials of the other groups $\left[F_{(3,53)}=2.94\right.$, $P=0.041]$. Post-hoc tests showed the Bi-9 group to have significantly more improvement than all other groups, whether compared with all three trials $(P<0.017$ for each) or to just the last two retest trials $(P<0.038$ for each) of the other groups. Differences in overnight improvement in error rate were not significant [ANOVA; $F_{(3,53)}=0.13, P=0.941$ ), although it should be noted that the error rate measure contains larger inherent variability than the performance speed measure.

There was also no significant relationship between the amount of initial improvement achieved across training and the subsequent amount of overnight improvement for either speed of accuracy in any group $(r<0.4 ; P>0.14)$, similar to previous findings (Walker et al. 2003b).

To summarize these data, it is apparent that there was significant overnight improvement for all groups, and thus, configurations of the sequential task demonstrating that delayed learning is not restricted to the simplest forms of such motor skills. The amount of overnight improvement was similar for the short five-element sequence, regardless of whether it required coordination within one hand (Uni-5, four fingers) or two hands (Bi-5, four fingers). However, increasing the sequence length to nine elements using one hand (Uni-9, four fingers), and especially performing the most complex nine-element bimanual task version, produced even greater overnight increases in skill speed.

\section{Within-sequence analysis of transition speed}

Whereas the overall measures of speed and accuracy exhibited clear overnight changes in performance, it was unclear whether there were more subtle, qualitative performance changes as well. To assess this possibility, we analyzed differences in transition speeds between each of the separate key press movements within each sequence before and after sleep. For example, in Group 1, performing the unimanual five-element sequence 4-1-3-2-4, there are four unique key-press transitions (a) from 4 to 1 , (b) from 1 to 3, (c) from 3 to 2, and (d) from 2 to 4 (see Materials and Methods).

When individual subjects' transition-speed profiles were analyzed, it was clear that, even within groups, subjects differed considerably in terms of which transitions were easiest (i.e., the fastest transition) and which were hardest (i.e., the slowest transition speed or problem point). This was the case in all groups. Similar intersubject variations have previously been reported (Sakai et al. 2003).

For each subject, we therefore identified prior to sleep, the fastest and slowest transition positions at posttraining (trials 1012). We then measured changes in speed at each of these same transitions positions (fastest and slowest) at retesting following the night of sleep, and asked whether both transition positions showed similar overnight improvement, or whether sleep selectively improved one of these transitions preferentially.

Interestingly, there was a clear dissociation in overnight improvement between the slowest and fastest transitions position across all groups [ANOVA (Fastest $\times$ Slowest) $F_{(1,53)}=19.8$, $P<0.0001$ ], with the slowest, most difficult transition (problem point) exhibiting a consistently large and significant improvement across all groups following sleep, averaging $17.8 \%$ $(P<0.001$ for each group), whereas no such improvement developed at the fastest transitions (mean improvement $=1.4 \%$; $P>0.12$ for each group). Thus, irrespective of the task characteristics, be it uni- or bimanual, short or long sequence, all groups showed a selective and similarly large overnight benefit at the slowest transition position, without any improvement at the fastest position. Group values and statistical analyses for each of these transitions are shown in Table 1A, and displayed in Figure 2. Representative single-subject examples for each of the four task configurations are shown in Figure 3.

These findings, by themselves, did not demonstrate that the selective improvement of the most difficult transition is a property of sleep-dependent consolidation. It might, for example, reflect a general property of motor sequence learning, occurring during training as well as during sleep-dependent consolidation. Alternatively, it might reflect consolidation processes occurring across periods of wake as well as sleep.

To determine whether the pattern of selective improvement seen overnight occurs during training, we repeated the analysis of slowest and fastest transition speeds for each of the four groups, but comparing transition speeds at the start of training to those at the end of training. Contrary to the pattern seen over-

Table 1A. Average group values for the slowest and fastest transitions pre- and post-sleep, together with the corresponding percent improvement overnight and the associated degree of significance

\begin{tabular}{|c|c|c|c|c|c|c|c|c|}
\hline \multirow[b]{2}{*}{ Group } & \multicolumn{4}{|c|}{ Slowest transition (problem point) } & \multicolumn{4}{|c|}{ Fastest transition (easy point) } \\
\hline & $\begin{array}{l}\text { Pre-sleep } \\
\text { speed (ms) }\end{array}$ & $\begin{array}{l}\text { Post-sleep } \\
\text { speed (ms) }\end{array}$ & $\%$ improv't & P-value & $\begin{array}{l}\text { Pre-sleep } \\
\text { speed (ms) }\end{array}$ & $\begin{array}{l}\text { Post-sleep } \\
\text { speed (ms) }\end{array}$ & $\%$ improv't & P-value \\
\hline Group 1 (Uni-5) & 303 & 247 & $18.5 \%$ & $<0.001$ & 198 & 191 & $3.5 \%$ & n.s. \\
\hline Group $2(\mathrm{Bi}-5)$ & 210 & 173 & $17.6 \%$ & $<0.001$ & 160 & 159 & $0.6 \%$ & n.s. \\
\hline Group 3 (Uni-9) & 328 & 260 & $20.7 \%$ & $<0.001$ & 165 & 166 & $-0.6 \%$ & n.s. \\
\hline Group 4 (Bi-9) & 490 & 397 & $19.0 \%$ & $<0.001$ & 161 & 163 & $-1.2 \%$ & n.s. \\
\hline
\end{tabular}




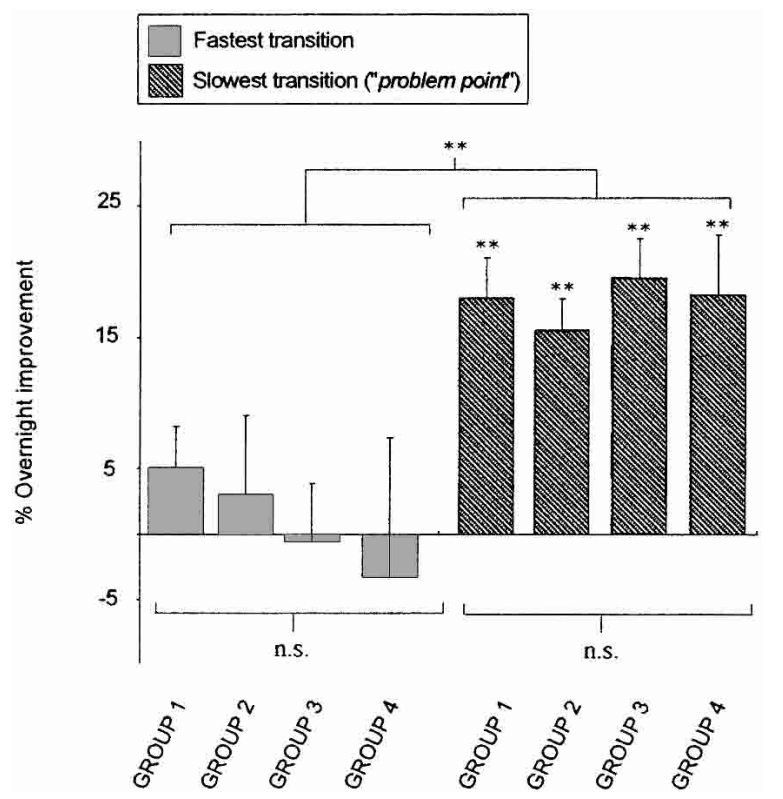

Figure 2. Group difference in the overnight percentage improvement in transition speed occurring at the fastest and slowest transition positions. Prior to sleep, the fastest transition positions and slowest transition positions at the end of training were identified for each subject in each group, and following a night of sleep, the percentage change at each of these same two transitions positions was measured. A clear dissociation in overnight improvement was evident, with all groups exhibiting a consistent and large improvement following sleep for the slowest transition (light gray bars, problem point), but no group showing significant improvement for the fastest transition (dark-hatched gray bars). Error bars, SEM; Asterisks represent significance $(P):\left({ }^{* *}\right)<0.005$; (n.s.) nonsignificant.

night, across training, all groups expressed large and significant improvements at both the slowest and fastest transitions (Table 1B). Specifically, there was an average $47 \%$ improvement during training for the slowest (problem point) transition $(<0.001$ for each group), and a 31\% improvement for the fastest transition ( $<0.005$ for all groups). Thus, the selective improvement of the slowest problem-point transition was specific to the overnight learning process, and was not seen during training.

To determine whether selective improvement could develop across periods of wake, an additional group of subjects $(n=12$; matched in age and gender) was trained on the unimanual fiveelement task in the morning (11 am $\pm 1 \mathrm{~h})$, and retested $8 \mathrm{~h}$ later. Consonant with previous reports (Fischer et al. 2002; Walker et al. 2002, 2003a; Korman et al. 2003), there was no significant change across the 8-h waking interval in the basic measures of performance speed, which improved just $4 \%$ $\left[\mathrm{t}_{(11)}=0.88, P=0.40\right]$, or accuracy, which decreased by $25 \%$ $\left[\mathrm{t}_{(11)}=0.55, P=0.59\right]$. When the within-sequence transition pro- files were analyzed, neither the fastest nor the slowest transitions showed significant changes in speed, with the slowest transition showing a $0.1 \%$ difference across the waking interval $\left[\mathrm{t}_{(11)}=0.39\right.$, $P=0.70]$, and the fastest transition actually slowing by an average of $7.1 \%\left[\mathrm{t}_{(11)}=1.06, P=0.30\right]$. From these data, it appears clear that no such selective improvement occurred in either the fastest or slowest transitions across $8 \mathrm{~h}$ of wake.

In summary, the overnight consolidation of motor-skill learning was associated with a selective improvement in keypress transitions that were slowest and most difficult prior to sleep, whereas transitions that were already fast prior to sleep showed no changes. This was in sharp contrast to the patterns of change seen across episodes of wake, where no significant changes occurred, or during initial training, where the slowest and the fastest transitions both show significant improvement.

\section{Discussion}

The current study focused on changes in the amount of overnight, sleep-dependent improvement in motor-skill learning when the task characteristics were modified across two dimensions: (1) the limb complexity-either unimanual or bimanual, and (2) the sequence length-either five elements or nine elements. We had previously reported that performance on a short, five-element unimanual task exhibits delayed improvements in speed and accuracy expressly across a night of sleep, and not across equivalent waking episodes (Walker et al. 2002). Our new results add to our previous findings (Walker et al. 2002, 2003a,b), and those of others (Fischer et al. 2002; Korman et al. 2003) in three important ways.

Firstly, they demonstrate that delayed, sleep-dependent learning is not limited, for example, to a simple motor sequence performed using the digits of one hand. Instead, delayed overnight learning is equally expressed when both the procedural memory load (sequence length) and movement complexities (number of hands involved) increase. Secondly, these data suggest that as the task becomes more complex, in our case with a combined demand for a longer motor sequence and bimanual coordination, the sleep-dependent gains actually increase. Finally, by examining more closely within-sequence reaction times, we have demonstrated that specific motor-sequence transitions that were most difficult prior to sleep are selectively improved across a night of sleep, whereas transition movements that were most effectively mastered prior to sleep are not subsequently enhanced overnight. This characteristic was consistent across all groups, irrespective of the task characteristics.

\section{Sleep-dependent learning and motor skill complexity}

Overnight learning was expressed across a variety of motor-skill levels, using both short and long sequences requiring coordinated movements either within one hand or between two. Thus, the neural mechanisms supporting delayed sleep-dependent enhancement can serve a range of learning demands. The finding of

Table 1B. Average group values for the slowest and fastest transitions at the start and end of training, together with the corresponding percent improvement across training and the associated degree of significance

\begin{tabular}{|c|c|c|c|c|c|c|c|c|}
\hline \multirow[b]{2}{*}{ Group } & \multicolumn{4}{|c|}{ Slowest transition (problem point) } & \multicolumn{4}{|c|}{ Fastest transition (easy point) } \\
\hline & $\begin{array}{l}\text { Training-start } \\
\text { speed (ms) }\end{array}$ & $\begin{array}{l}\text { Training-end } \\
\text { speed (ms) }\end{array}$ & $\%$ improv't & P-value & $\begin{array}{l}\text { Training-start } \\
\text { speed (ms) }\end{array}$ & $\begin{array}{l}\text { Training-end } \\
\text { speed (ms) }\end{array}$ & $\%$ improv't & P-value \\
\hline Group 1 (Uni-5) & 403 & 283 & $29.7 \%$ & $<0.001$ & 270 & 203 & $24.8 \%$ & 0.002 \\
\hline Group 2 (Bi-5) & 373 & 191 & $48.8 \%$ & $<0.001$ & 260 & 188 & $28.5 \%$ & 0.001 \\
\hline Group 3 (Uni-9) & 504 & 253 & $53.4 \%$ & $<0.001$ & 245 & 191 & $22.0 \%$ & 0.005 \\
\hline Group 4 (Bi-9) & 908 & 400 & $55.9 \%$ & $<0.001$ & 335 & 172 & $48.7 \%$ & $<0.001$ \\
\hline
\end{tabular}


A) GROUP 1 Uni-5

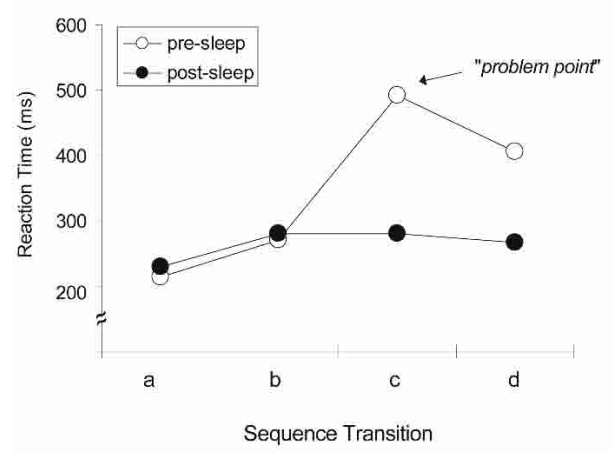

C) GROUP 3 Uni-9

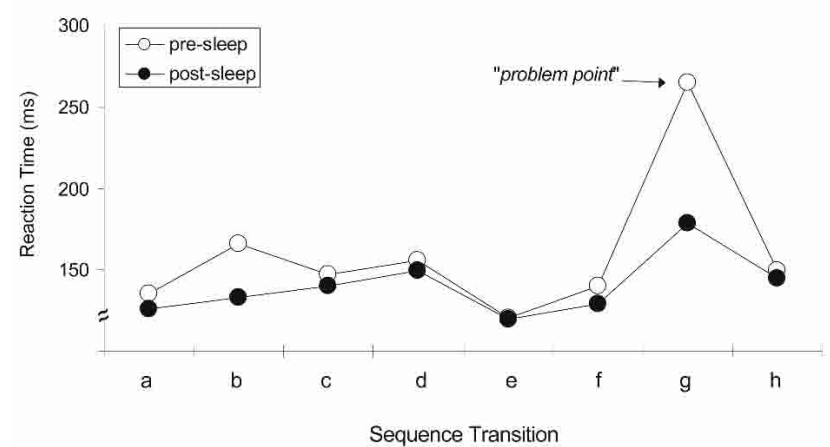

B) GROUP 2 Bi-5

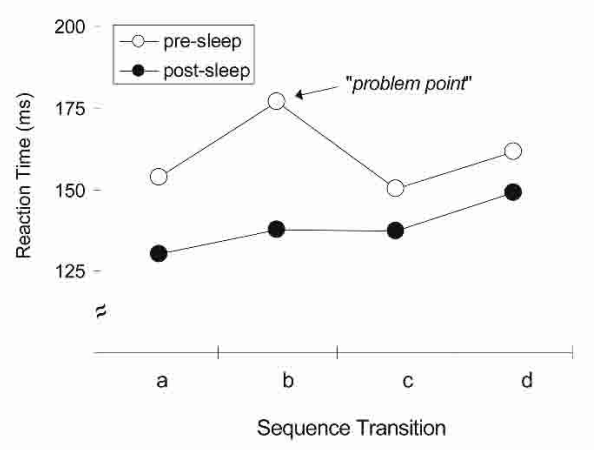

D) GROUP $4 \quad \mathrm{Bi}-9$

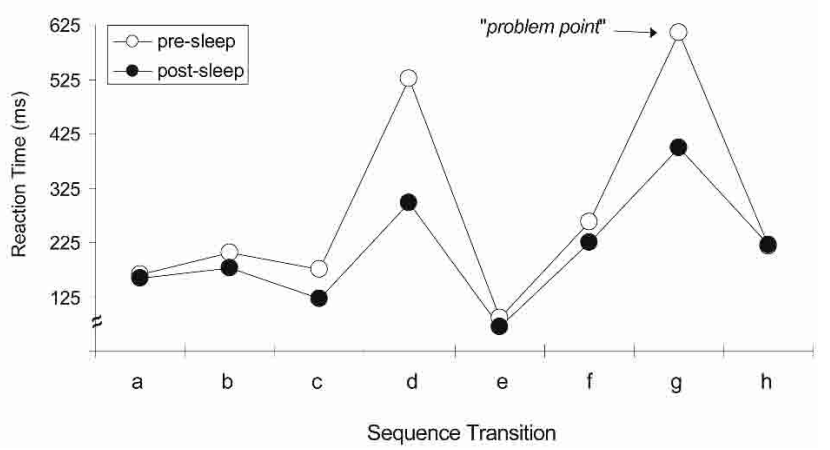

Figure 3. Representative single-subject examples of the pre- and postsleep transition profiles within sequences for each of the four groups. For an individual subject, in each of the groups (1-4, $A, B, C, D$, respectively), the pre-sleep transition profile $(0)$ and the post-sleep transition profile $(\bullet)$ was calculated within each sequence. Pre-sleep profiles demonstrated considerable variability, with certain transitions being particularly slow (most difficult; problem points), whereas other transitions appear to be relatively rapid (easy). Yet, a prominent feature following a night of sleep, independent of the group/task, was a specific reduction (improvement) in the speed of the slowest problem-point transition, whereas the faster transitions prior to sleep showed a lack of consistent improvement following sleep.

delayed overnight improvement across a range of task difficulty only strengthens the hypothesis that a similar sleep-dependent mechanism underlies the mastering of many real-life complex motor skills, from learning to play musical instruments and mastering the coordinated movements in sports to refining surgical skills and learning motor patterns for intricate cognitive operations such as speech articulation. Moreover, it is perhaps no longer an unreasonable extrapolation to suggest that sleep plays an important role in the functional recovery of motor skills following brain insults such as stroke.

\section{Differential degrees of improvement and motor skill complexity}

Although intense investigation has focused on the nature and complexity of practice-dependent motor-skill learning (for reviews, see Willingham 1998; Hikosaka et al. 2002), this study is the first to have examined subsequent differences in delayed learning across sleep. Whereas all groups showed significant overnight performance improvements, comparisons between groups suggested that the beneficial effects of a night of sleep were subtly different across these different task-complexity levels. Interestingly, and partially contrary to our initial hypothesis, little difference in overnight improvement was found between groups learning unimanual and bimanual five-element sequences (Uni-5 and Bi-5, respectively), with only a modestly greater improvement in the group learning the unimanual nineelement sequence (Uni-9), even though there were changes in both the memory load and the degree of between-limb coordination. However, the most complex version of the task, the bimanual nine-element sequence (Bi-9), showed a considerably larger change in performance speed, with the amount of overnight improvement being 63\% greater than that of Uni-5 and Bi-5 groups. It should be noted, however, that error rate did not decrease as much in Group 4, and thus, a speed accuracy trade off cannot be discounted, although no significant difference was found between error rates across all groups.

Mechanistically, we consider two possible, nonmutually exclusive explanations for the larger overnight improvement in Group 4. The first is that alterations in the magnitude of sleepdependent learning are determined by the different task characteristics, but in a nonlinear, composite manner, meaning that changing one axis of task difficulty alone (i.e., from a unimanual to bimanual, or from a short to long sequence) does not trigger greater overnight learning. Instead, both of these characteristics have to be increased together (i.e., a bimanual, long-sequence configuration) for a significant difference to be seen.

The second possibility, and one which we favor, is a more mechanistic determinant of differences in learning, relating to the degree of plasticity. Although task characteristics were different in groups $1-3$, the total number of digits used to perform the task was identical-four fingers-in each of the groups. In contrast, subjects in Group 4 were required to utilize not only cooperative movements between hands, and cope with a high memory load, but also had to use all eight digits. As such, the 
extent of cerebral networks representing this memory would be considerably greater, as would be the ensuing degree of potential plasticity.

The latter hypothesis is supported by several lines of physiological evidence, in animals and humans, indicating that bimanual complex movements engage somewhat different neural systems in cortical (Gordon et al. 1998; Kazennikov et al. 1999; Jancke et al. 2000; Toyokura et al. 2002; Nair et al. 2003) and subcortical (Wannier et al. 2002) regions, as well as in the cerebellum (Tracy et al. 2001). Furthermore, on the basis of the known somatotopy of the motor cortex and cerebellum (Rijntjes et al. 1999; Kurth et al. 2000; Beisteiner et al. 2001), the size of networks involved in controlling movements appears to vary as a function of the number of digits used in task performance. Taken together, these findings suggest that a bimanual task, incorporating all eight fingers, would not only engage a more extensive cortical, subcortical, and cerebellar system, but also demand a greater area of involvement within these regions in terms of coding the memory representation, perhaps proportional to the increased number of digits used. As such, the network size of the memory representation, and thus, the potential for sleepdependent synaptic plasticity, would be considerably greater in the Bi-9 task using cooperative movements across eight fingers. This hypothesis is corroborated by the considerably greater amount of overnight improvement in speed in the Bi-9 group, relative to the other three groups using only four fingers. Future functional anatomical studies of the sleep-dependent differences between these task configurations will add much to such conjectures.

\section{Differential degrees of improvement and within-sequence transitions}

A host of previous reports have described the appearance of uneven spacing in the timing of key-press transitions within motorsequence patterns-a phenomenon that has been termed "chunking" (Rosenbaum et al. 1983; Verwey and Dronkert 1996; Graybiel 1998; Sakai et al. 2003; Stöcker and Hoffmann 2004). The implication is that large motor-sequence patterns are initially learned in smaller subsequences, each of which is processed as a single memory unit, indicative of a motor-sequence learning hierarchy. In a recent study, Sakai et al. (2003) reported evidence of chunking or uneven temporal sequence performance at different positions of the sequence in different subjects, even though they were learning the same sequence. This would suggest that individual subjects chunk the same sequence differently during practice-dependent learning.

We discovered similar evidence of subject-specific chunking of a sequence, punctuated by distinct slowing at individual problem points within the sequence. Remarkably, following a night of sleep and its associated learning benefits, the within-sequence transition profile was radically different, with subjects' slowest transitions (most difficult) being specifically improved relative to their fastest transitions. Thus, although the slowest problempoint transitions improved in speed overnight by an average of $19 \%$, the fastest transitions showed only 1\% improvement, and the gap between fastest and slowest transitions was decreased by over a third. Thus, transitions within the motor sequence that were initially most difficult appeared to be preferentially improved overnight. That this is not due to a ceiling effect is made clear by analysis of data from a previous study (Walker et al. 2003b, Group 3), where subjects received twice the amount of initial training on Day 1 in the Uni-5 protocol. In this case, the fastest transition speeds measured after a night of sleep averaged $145 \mathrm{msec},>30 \%$ faster than the one seen in Group 1 in the current study.
This pattern of preferential overnight improvement of the slowest problem point transition was not evident in an additional group of subjects trained in the morning and retested after an 8-h waking interval, demonstrating no significant improvement in either the fastest or slowest transitions. Moreover, this preferential pattern of improvement was also absent across initial training in all four groups, with the slowest and fastest transitions showing similar improvements.

On the basis of these additional comparisons, it is clear that the selective improvement of the most difficult key-press transitions does not develop either during initial training or across an extensive period of posttraining wake. From these findings, we therefore consider the most reasonable explanation for the preferential overnight improvement of the most difficult transition as being specifically related to the process of sleep-dependent memory enhancement itself (Fischer et al. 2002; Walker et al. 2002, 2003a,b).

In the context of the chunking framework, it is interesting to speculate that the sleep-dependent learning process provides a greater degree of motor-program automatization, resulting not only in faster transitions within the sequence, but more uniform transitions as well. If correct, this would indicate that the sleepdependent learning mechanism may involve amalgamating disparate subsequence memory units into a larger single memory representation. Furthermore, this characteristic overnight improvement at the slower problem points, regardless of sequence length or cooperative movement complexity, suggests that the normalization of key-press transition speeds is a pervasive property of the sleep-dependent learning process.

\section{Conclusions}

When the degree of motor-skill complexity, both in terms of memory load (a nine-element sequence vs. a five-element sequence) and extent of movement coordination (bimanual vs. unimanual), was altered, overnight learning occurred across all levels of task complexity, suggesting the sleep-dependent memory enhancement is not limited to simple motor-skill learning. In addition, although overnight improvement was common across all task configurations, the most complex combination of motor-skill complexity (the bimanual nine-element task) exhibited significantly greater delayed benefit, with performance speed being more than twice that of the simpler unimanual fiveelement task. This effect may be mediated by the degree of potential plasticity, as twice the number of digits were used to encode this memory representation in the bimanual nine-element group.

When performance profiles within sequences were examined, pre-sleep transition speeds were seen to be uneven, with some key-press transitions appearing to be easy (fast) and others problematic (slow), as if the entire sequence was being parsed into smaller subsequences. After a night of sleep, however, these problematic slow transitions were preferentially improved, suggesting that the sleep-dependent learning process involves the unification of smaller memory units into one single memory element.

In this study, we asked how sleep-dependent overnight improvement on a simple finger-tapping motor-sequence task varied locally, within the motor sequence (transition speeds), and globally, with the complexity of the task (number of key-presses, fingers, and hands required). In each case, we found that the fundamental characteristics of motor-sequence learning that were the most difficult (be it the complexity of the Bi-9 sequence or, on a smaller scale, the slowest key-press transitions) showed the greatest overnight improvement, and raises the possibility that this reflects a more general strategy used by the brain during sleep-dependent learning and memory processing. 


\section{Materials and Methods}

\section{Participants}

A total of 57 healthy right-handed subjects between the ages of 18 and 31 [mean $22.5 \pm 1.22$ (SD); 40 females] were assigned in a balanced order to four different groups (see below). Subjects had no prior history of drug or alcohol abuse, neurological, psychiatric, or sleep disorders, and were maintaining a regular sleep schedule. Subjects were instructed to be drug-, alcohol-, and caffeine-free for $24 \mathrm{~h}$ prior to and during the study period. All studies were approved by the local human studies committee, and all subjects provided written informed consent.

\section{Motor-skill task}

Four different forms of the sequential finger-tapping task were used, corresponding to the four different experimental groups (Groups 1-4; see Fig. 4 and below for details). Subjects performed the task using a standard PC keyboard, typing the designated sequence repeatedly and as quickly and as accurately as possible for a period of $30 \mathrm{sec}$ (Walker et al. 2002, 2003b). The numeric sequence was displayed at the top of the screen at all times to exclude any working memory component to the task. Each key press produced a white dot on the screen, forming a row from left to right, rather than the number itself, so as not to provide accuracy feedback, with the program recording the key-press response.

Training consisted of 12,30 -sec trials with 30 -sec rest periods between trials, thus lasting a total of $12 \mathrm{~min}$. The performance from the first trial of the training session was taken as the baseline measure, whereas the average scores from the final three trials were taken as the posttraining performance. At the subsequent retest following sleep, subjects performed three 30 -sec trials of the same sequence separated by 30 -sec rest periods, with the scores again being averaged.

\section{Performance measures}

Performance was evaluated using overall trial-performance measures, as well as subtler measures of within-sequence performance. Overall measures were the same two variables used in our previous studies, those being (1) the number of correctly typed sequences per 30-sec trial (Speed), and (2) the number of errors made relative to the number of correctly typed sequences per 30-sec trial (Accuracy).

The more detailed, within-sequence measure assessed the time intervals between successive key presses within the sequence (Transition Speed), for example, the interval between pressing the first and second key of the sequence, or between the fourth and fifth key (see Fig. 4). This measure is therefore able to provide a temporal profile of the key-press transition speed for each individual subject performing the same sequence.

For measuring overnight improvement, average performance on the last three of the 12 training trials was compared with average performance on the three trials at retest. For measuring the transition speed changes during initial training, the average performance on the first two trials of training (trainingstart) was compared with the average performance on the last two trials of training (training-end).

\section{Experimental groups}

The 57 subjects were assigned to four experimental groups, all of which were trained at 1 PM on Day 1 , and following one night of sleep, were retested at $1 \mathrm{PM}$ on Day 2 ( $24 \mathrm{~h}$ posttraining).

\section{Group 1: Unimanual five-element (Uni-5, $n=15$ )}

Subjects were instructed to press four numeric keys on a standard computer keyboard with the fingers of their left hand, repeating the five-element sequence, $4-1-3-2-4$, as quickly and as accurately as possible, for periods of $30 \mathrm{sec}$. Thus, four fingers on one hand were used (Fig. 4A).

Group 2: Bimanual five-element (Bi-5, $n=15$ )

Subjects again had to press four numeric keys on the keyboard, but now were required to use fingers from both hands, repeating the five-element sequence, 4-6-1-7-4, as quickly and as accurately

\section{Learning \& Memory}

www.learnmem.org 
as possible, for a period of $30 \mathrm{sec}$. Thus, four fingers were used; two from each hand (Fig. 4B).

\section{Group 3: Unimanual nine-element (Uni-9, $n=13$ )}

Subjects pressed four numeric keys on the keyboard with the fingers of their left hand as in Group 1, but repeated a longer nine-element sequence, 4-1-3-2-1-3-2-1-4, as quickly and as accurately as possible, for periods of $30 \mathrm{sec}$. But only four fingers on one hand were used (Fig. 4C).

\section{Group 4: Bimanual nine-element (Bi-9, $n=14$ )}

Subjects had to press eight numeric keys on the keyboard, using four fingers from each hand, repeating the nine-element sequence, 4-6-1-7-3-2-5-8-4, as quickly and as accurately as possible. In this case, eight fingers, four on each hand, were used (Fig. 4D).

\section{Sleep quality and alertness}

At each training and retest session, subjects completed the Stanford Sleepiness Scale, a standard measure of subjective alertness (Hoddes et al. 1973). As expected, there were no significant differences in the ratings of alertness, either between groups $\left[F_{(3,53)}=0.119, P=0.948\right]$ or between the $2 \mathrm{~d}$ within any of the groups $(P>0.39)$. The amount of overnight sleep obtained by subjects in each group was documented with sleep logs, and averaged $7.6 \mathrm{~h}( \pm 1.15 ; \mathrm{SD})$ across the experimental night. There was no significant difference between groups in the amount of reported sleep $\left[F_{(3,53)}=1.774, P=0.163\right]$.

\section{Statistical analysis}

Analyses were carried out with one-way and two-way ANOVAs with post-hoc comparisons, paired and two-sample two-tailed Student's t-test, and Pearson's correlation coefficient.

\section{Acknowledgments}

This work was supported by grants from the National Institutes of Health (MH069935; MH48832; MH65292, and MH67754), the National Science Foundation (BCS-0121953), the Japanese Foundation for Aging and Health Research on Cancer Prevention and Health Services, and the Japanese Health and Labour Sciences Research Grant program. We thank Alex Morgan for technical assistance.

\section{References}

Andres, F.G., Mima, T., Schulman, A.E., Dichgans, J., Hallett, M., and Gerloff, C. 1999. Functional coupling of human cortical sensorimotor areas during bimanual skill acquisition. Brain 122: 855-870.

Atienza, M., Cantero, J.L., and Stickgold, R. 2004. Posttraining sleep enhances automaticity in perceptual discrimination. J. Cogn. Neurosci. 16: 53-64.

Beisteiner, R., Windischberger, C., Lanzenberger, R., Edward, V., Cunnington, R., Erdler, M., Gartus, A., Streibl, B., Moser, E., and Deecke, L. 2001. Finger somatotopy in human motor cortex. Neuroimage 13: 1016-1026.

De Weerd, P., Reinke, K., Ryan, L., McIsaac, T., Perschler, P., Schnyer, D., Trouard, T., and Gmitro, A. 2003. Cortical mechanisms for acquisition and performance of bimanual motor sequences. Neuroimage 19: 1405-1416.

Fischer, S., Hallschmid, M., Elsner, A.L., and Born, J. 2002. Sleep forms memory for finger skills. Proc. Natl. Acad. Sci. 99: 11987-11991.

Gaab, N., Paetzold, M., Becker, M., Walker, M.P., and Schlaug, G. 2004. The influence of sleep on auditory learning-A behavioral study. Neuroreport 15: 731-734.

Gais, S., Plihal, W., Wagner, U., and Born, J. 2000. Early sleep triggers memory for early visual discrimination skills. Nat. Neurosci. 3: 1335-1339.

Gerloff, C. and Andres, F.G. 2002. Bimanual coordination and interhemispheric interaction. Acta. Psychol. (Amst) 110: 161-186.

Gordon, A.M., Lee, J.H., Flament, D., Ugurbil, K., and Ebner, T.J. 1998. Functional magnetic resonance imaging of motor, sensory, and posterior parietal cortical areas during performance of sequential typing movements. Exp. Brain Res. 121: 153-166.

Grafton, S.T., Hazeltine, E., and Ivry, R.B. 2002. Motor sequence learning with the nondominant left hand. A PET functional imaging study. Exp. Brain Res. 146: 369-378.

Graybiel, A.M. 1998. The basal ganglia and chunking of action repertoires. Neurobiol. Learn. Mem. 70: 119-136.

Hikosaka, O., Nakamura, K., Sakai, K., and Nakahara, H. 2002. Central mechanisms of motor skill learning. Curr. Opin. Neurobiol. 12: 217-222.
Hoddes, E., Zarcone, V., Smythe, H., Philips, R., and Dement, W.C. 1973. Quantification of sleepiness: A new approach. Psychophysiology 10: $431-436$.

Jancke, L., Peters, M., Himmelbach, M., Nosselt, T., Shah, J., and Steinmetz, H. 2000. fMRI study of bimanual coordination. Neuropsychologia 38: 164-174.

Karni, A., Tanne, D., Rubenstein, B.S., Askenasy, J.J., and Sagi, D. 1994. Dependence on REM sleep of overnight improvement of a perceptual skill. Science 265: 679-682.

Kazennikov, O., Hyland, B., Corboz, M., Babalian, A., Rouiller, E.M., and Wiesendanger, M. 1999. Neural activity of supplementary and primary motor areas in monkeys and its relation to bimanual and unimanual movement sequences. Neuroscience 89: 661-674.

Korman, M., Raz, N., Flash, T., and Karni, A. 2003. Multiple shifts in the representation of a motor sequence during the acquisition of skilled performance. Proc. Natl. Acad. Sci. 100: 12492-12497.

Kurth, R., Villringer, K., Curio, G., Wolf, K.J., Krause, T., Repenthin, J., Schwiemann, J., Deuchert, M., and Villringer, A. 2000. fMRI shows multiple somatotopic digit representations in human primary somatosensory cortex. Neuroreport 11: 1487-1491.

Miller, G.A. 1956. The magical number seven plus or minus two: Some limits on our capacity for processing information. Psychol. Rev. 63: 81-97.

Muller, R.A., Kleinhans, N., Pierce, K., Kemmotsu, N., and Courchesne, E. 2002. Functional MRI of motor sequence acquisition: Effects of learning stage and performance. Brain Res. Cogn. Brain Res. 14: 277-293.

Nair, D.G., Purcott, K.L., Fuchs, A., Steinberg, F., and Kelso, J.A. 2003. Cortical and cerebellar activity of the human brain during imagined and executed unimanual and bimanual action sequences: A functional MRI study. Brain Res. Cogn. Brain Res. 15: 250-260.

Rijntjes, M., Buechel, C., Kiebel, S., and Weiller, C. 1999. Multiple somatotopic representations in the human cerebellum. Neuroreport 10: 3653-3658.

Robertson, E.M., Pascual-Leone, A., and Press, D.Z. 2004. Awareness modifies the skill-learning benefits of sleep. Curr. Biol. 14: 208-212.

Rosenbaum, D.A., Kenny, S.B., and Derr, M.A. 1983. Hierarchical control of rapid movement sequences. J. Exp. Psychol. Hum. Percept. Perform. 9: $86-102$.

Sakai, K., Kitaguchi, K., and Hikosaka, O. 2003. Chunking during human visuomotor sequence learning. Exp. Brain Res. 152: 229-242.

Smith, C. 1995. Sleep states and memory processes. Behav. Brain Res. 69: $137-145$.

Smith, C. and MacNeill, C. 1994. Impaired motor memory for a pursuit rotor task following Stage 2 sleep loss in college students. J. Sleep Res. 3: 206-213.

Stickgold, R., James, L., and Hobson, J.A. 2000a. Visual discrimination learning requires sleep after training. Nat. Neurosci. 3: 1237-1238.

Stickgold, R., Whidbee, D., Schirmer, B., Patel, V., and Hobson, J.A. 2000b. Visual discrimination task improvement: A multi-step process occurring during sleep. J. Cogn. Neurosci. 12: 246-254.

Stickgold, R., Hobson, J.A., Fosse, R., and Fosse, M. 2001. Sleep, learning, and dreams: Off-line memory reprocessing. Science 294: 1052-1057.

Stöcker, C. and Hoffmann, J. 2004. The ideomotor principle and motor sequence acquisition: Tone effects facilitate movement chunking. Psychol. Res. 68: 126-137.

Toyokura, M., Muro, I., Komiya, T., and Obara, M. 2002. Activation of pre-supplementary motor area (SMA) and SMA proper during unimanual and bimanual complex sequences: An analysis using functional magnetic resonance imaging. J. Neuroimaging 12: 172-178.

Tracy, J.I., Faro, S.S., Mohammed, F.B., Pinus, A.B., Madi, S.M., and Laskas, J.W. 2001. Cerebellar mediation of the complexity of bimanual compared to unimanual movements. Neurology 57: 1862-1869.

Verwey, W.B. and Dronkert, Y. 1996. Practicing a structured continuous key-pressing task: Motor chunking or rhythm consolidation? J. Mot. Behav. 28: 71-79.

Walker, M.P. 2004. A refined model of sleep and the time course of memory formation. Behav. Brain Sci. (in press).

Walker, M.P., Brakefield, T., Morgan, A., Hobson, J.A., and Stickgold, R. 2002. Practice with sleep makes perfect: Sleep dependent motor skill learning. Neuron 35: 205-211.

Walker, M.P., Brakefield, T., Hobson, J.A., and Stickgold, R. 2003a. Dissociable stages of human memory consolidation and reconsolidation. Nature 425: 616-620.

Walker, M.P., Brakefield, T., Seidman, J., Morgan, A., Hobson, J.A., and Stickgold, R. 2003b. Sleep and the time course of motor skill learning. Learn. Mem 10: 275-284.

Wannier, T., Liu, J., Morel, A., Jouffrais, C., and Rouiller, E.M. 2002. Neuronal activity in primate striatum and pallidum related to bimanual motor actions. Neuroreport 13: 143-147.

Willingham, D.B. 1998. A neuropsychological theory of motor skill learning. Psychol. Rev. 105: 558-584.

Received March 1, 2004; accepted in revised form September 8, 2004. 


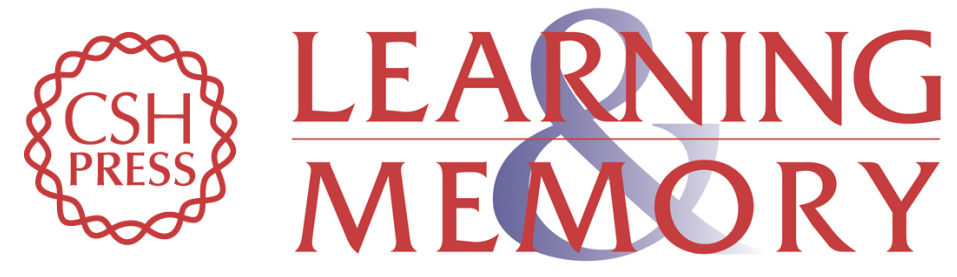

\section{Sleep-dependent learning and motor-skill complexity}

Kenichi Kuriyama, Robert Stickgold and Matthew P. Walker

Learn. Mem. 2004, 11:

Access the most recent version at doi:10.1101//m.76304

References This article cites 39 articles, 6 of which can be accessed free at: http://learnmem.cshlp.org/content/11/6/705.full.html\#ref-list-1

License

Email Alerting Receive free email alerts when new articles cite this article - sign up in the box at the Service top right corner of the article or click here. 